\title{
anlotinib alters tumor immune microenvironment by downregulating PD-L1 expression on vascular endothelial cells
}

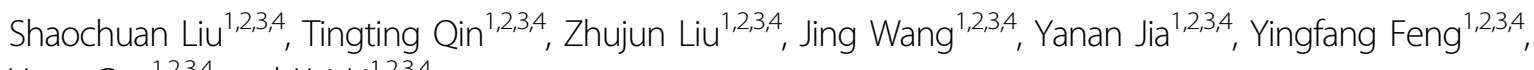
Yuan Gao ${ }^{1,2,3,4}$ and Kai Li, $i^{1,2,3,4}$

\begin{abstract}
Aberrant vascular network is a hallmark of cancer. However, the role of vascular endothelial cells (VECs)-expressing PDL1 in tumor immune microenvironment and antiangiogenic therapy remains unclear. In this study, we used the specimens of cancer patients for immunohistochemical staining to observe the number of PD-L $1^{+} \mathrm{CD} 34^{+} \mathrm{VECs}$ and infiltrated immune cells inside tumor specimens. Immunofluorescence staining and flow cytometry were performed to observe the infiltration of $\mathrm{CD}^{+} \mathrm{T}$ cells and FoxP3 ${ }^{+} \mathrm{T}$ cells in tumor tissues. Here, we found that PD-L1 expression on VECs determined $\mathrm{CD}^{+}{ }^{+}$cells', FoxP3 ${ }^{+} \mathrm{T}$ cells' infiltration, and the prognosis of patients with lung adenocarcinoma. Anlotinib downregulated PD-L1 expression on VECs through the inactivation of AKT pathway, thereby improving the ratio of CD8/FoxP3 inside tumor and remolding the immune microenvironment. In conclusion, our results demonstrate that PD-L1 high expression on VECs inhibits the infiltration of CD8 ${ }^{+} \mathrm{T}$ cells, whereas promotes the aggregation of FoxP3 ${ }^{+} \mathrm{T}$ cells into tumor tissues, thus becoming an "immunosuppressive barrier". Anlotinib can ameliorate the immuno-microenvironment by downregulating PD-L1 expression on VECs to inhibit tumor growth.
\end{abstract}

\section{Introduction}

The aberrant vasculature, one of hallmarks of cancer, helps neoplasms to escape from immune attack in tumor microenvironment. In specific speaking, the abnormal vascular network will constitute an immunosuppressive environment and inhibit the infiltration of effector $\mathrm{T}$ cells including $\mathrm{CD}^{+}$cytotoxic $\mathrm{T}$ lymphocytes that are the key effectors of antitumor immunity ${ }^{1,2}$.

Furthermore, aberrant vascular endothelial cells (VECs) can also participate in immune inhibition and suppress the function of immune cells in tumor microenvironment by expressing inhibitory receptors such as FasL, VACM-1, PD-L1 (program death ligand 1) and CD73, etc ${ }^{3,4}$. PD-L1

\footnotetext{
Correspondence: Kai Li (likai_fnk5@126.com)

${ }^{1}$ Tianjin Medical University Cancer Institute and Hospital, National Clinical

Research Center for Cancer, Tianjin, China

${ }^{2}$ Key Laboratory of Cancer Prevention and Therapy, Tianjin, China

Full list of author information is available at the end of the article

These authors contributed equally: Shaochuan Liu, Tingting Qin

Edited by H.-U. Simon
}

is one of the ligands of PD-1 and wildly expressed on various cells (such as immune cells, non-hematopoietic cells and tumor cells, etc.) in the tumor microenvironment ${ }^{5}$. As a variety of targeted drugs against PD-1/PD-L1 have obtained good results in many clinical trials, the PD1/PD-L1 pathway has become the hotspots of study. It has been confirmed that PD-L1 expression on VECs enhances the inhibitory function of FoxP3 ${ }^{+}$Treg cells $^{6}$ and declines the function of $\mathrm{CD}^{+} \mathrm{T}$ cells in various autoimmune diseases $^{7,8}$. However, it is still poorly understood about the detail of immune-modulatory effects of PD-L1 expression on VECs in tumor microenvironment.

According to the previous literature ${ }^{9}$, proinflammatory signaling may facilitate PD-L1 expression on VECs and decrease the efficacy of immunotherapies. Some studies ${ }^{10,11}$ suggest that the expression of PD-L1 on VECs is upregulated by increased IFN- $\gamma$ secreted after antiangiogenic therapy. According to these studies, we reasonably speculate that the expression of PD-L1 on VECs

\section{(c) The Author(s) 2020}

(c) (i) Open Access This article is licensed under a Creative Commons Attribution 4.0 International License, which permits use, sharing, adaptation, distribution and reproduction cc) in any medium or format, as long as you give appropriate credit to the original author(s) and the source, provide a link to the Creative Commons license, and indicate if changes were made. The images or other third party material in this article are included in the article's Creative Commons license, unless indicated otherwise in a credit line to the material. If material is not included in the article's Creative Commons license and your intended use is not permitted by statutory regulation or exceeds the permitted use, you will need to obtain permission directly from the copyright holder. To view a copy of this license, visit http://creativecommons.org/licenses/by/4.0/. 
will participate in antiangiogenic therapy and even affect the efficacy.

Anlotinib, a novel orally administered RTK inhibitor ${ }^{12}$, has showed promising efficacy in several clinical trials via inhibiting activation of various pro-angiogenic signals (VEGFR, PDGFR, and FGFR, etc.) ${ }^{13,14}$. However, the effect of anlotinib in tumor immune microenvironment remains largely unclear. Thus, this study aims to investigate the effects and underlying mechanism of anlotinib on PD-L1 expression in tumor VECs and tumor immune microenvironment.

We have demonstrated in this study that anlotinib can inhibit PD-L1 expression on VECs so as to break through the "immune tolerance barrier", promote $\mathrm{CD} 8^{+} \mathrm{T}$ cells infiltration, improve the balance of CD8/FoxP3. This work provides new insights into the effects of antiangiogenic therapy on tumor immune microenvironment, which is mediated by the change in the expression of PDL1 on VECs. In addition, this work reveals a new evidence and proposes a possible mechanism by which anlotinib affects the immune microenvironment to inhibit tumor cell proliferation.

\section{Materials and methods}

\section{Cell culture and reagents}

Human umbilical vein endothelial cells (HUVECs) and A549 were obtained from Peking union medical college cell bank; immortalized mouse brain endothelial cell lines (bEnd.3) were obtained from Nankai University; mouse retinal microvascular endothelial cells (MRMECs) were gifts from Tianjin medical university eye hospital; MC38 cells, B16 cells and B16-OVA cells were obtained from Dr. Yuan at Tianjin Medical University Cancer Institute and Hospital. All cell lines were performed cell line authentication before receipt, and all of the cell lines were passaged for no $>6$ months. Hypoxia $\left(<1 \% \mathrm{O}_{2}\right)$ was induced by a modular incubator chamber (Billups-Rothenberg). Bevacizumab (H0180801, Roche) was purchased from Roche company. Anlotinib and anti-PD-L1 drug were gifts from Nanjing Chia Tai Tianqing Company. Endothelial cells and B16 cells were grown using DMEM medium (Lonza) containing 10\% fetal bovine serum (FBS). MC38 cells were grown with RMPI 1640 (Thermo Flasher scientific) containing 10\% FBS. All cells were starved for $12 \mathrm{~h}$ before being stimulated by cytokines or the supernatants of tumor cells.

\section{Western blots analysis}

HUVECs, bEnd.3, and MEMRC were stimulated by the supernatant medium of A549 cells, vascular endothelial cell growth factor A (VEGFA) or cultured under hypoxia condition etc. for $24 \mathrm{~h}$. Protein samples were collected in ice-cold $1 \times$ RIPA buffer containing a protease inhibitor cocktail (Thermo Flasher scientific) and were separated using $10 \%$ sodium dodecyl sulphate-polyacrylamide gel electrophoresis. The proteins were transferred to polyvinylidenedifluoride membranes (Roche Molecular Biochemicals, Quebec, Canada), blocked with 5\% bovine serum albumin for $1 \mathrm{~h}$, and then incubated overnight at $4^{\circ} \mathrm{C}$ with appropriate primary antibodies. We used the primary antibodies including: PD-L1 (ab213524, Abcam), AKT (4691, Cell Signaling Technology), p-AKT (4060, Cell Signaling Technology), ERK (4695, Cell Signaling Technology), p-ERK (4370, Cell Signaling Technology), $\beta$-actin (8456, Cell Signaling Technology), PI3k (4255, Cell Signaling Technology), p-PI3K (4228, Cell Signaling Technology). The blots were further incubated with conjugated secondary antibodies and washed 3 times in TBST for 5 minutes each time. The blots were further incubated with HRP-conjugated secondary antibodies and developed with the ECL System (Millipore).

\section{Endothelial cells-T cells co-cultures}

HUVECs were pre-incubated with the supernatant conditioned medium (CM) of A549 cells for $48 \mathrm{~h}$ before co-culturing with PBMC. PBMC were extracted from normal blood of healthy volunteers and treated with $2.5 \mu \mathrm{g} / \mathrm{ml}$ anti-CD3 (OKT3, eBioscience) antibody and IL$2(100 \mathrm{U} / \mathrm{ml})$ in order to stimulate the activation of $\mathrm{T}$ cells. After $72 \mathrm{~h}$ of T-cell activation, PBMC were incubated with A549 CM-treated HUVEC cells at a 1:1 ratio for $48 \mathrm{~h}$. Different inhibitors or drugs, such as antiPD-L1 drugs $(10 \mu \mathrm{g} / \mathrm{ml})$, anti-VEGF antibody $(10 \mu \mathrm{g} / \mathrm{ml})$, and anlotinib $(1 \mu \mathrm{M})$ were added to the mix. The proliferation of $\mathrm{T}$ cells was measured by flow cytometric measurement of Ki67.

\section{Paraffin section of human tumor}

All paraffin sections of patient's tumor tissues were obtained from Tianjin Medical University Cancer Institute and Hospital. We collected all sections from patients with colon $(n=50)$, renal $(n=32)$ and lung cancers (primary and metastatic tumor samples from 106 patients with lung adenocarcinoma with treatment-naive stage I-IV) for immunohistochemical staining. All patients underwent surgery or treatment at Tianjin Medical University Cancer Institute and Hospital between 2011 and 2018. The clinical data of the patients collected included: age at diagnosis, gender, smoking history, gene mutation status, and clinical stage. All clinical parameters were retrieved according to the electronic medical record. The study was approved by the Tianjin Medical University Cancer Institute and Hospital's Ethics Committee and informed consents were obtained from all patients.

\section{Quantitation of TILs (tumor-infiltrating lymphocytes)}

Paraffin-embedded patient tumor tissue sections were placed at $70^{\circ} \mathrm{C}$ for $1 \mathrm{~h}$ or overnight, and immersed in 
xylene to remove paraffin, rehydrated in a continuous alcohol grading and washed in water for $5 \mathrm{~min}$. Sections were placed in citrate or ethylenediaminetetraacetic acid (depending on the antibody), and antigen retrieval were performed at $130^{\circ} \mathrm{C}$ for $2 \mathrm{~min}$. After being blocked with $3 \%$ hydrogen peroxide and normal goat serum, the sections were incubated with primary antibody at $4^{\circ} \mathrm{C}$ overnight, followed by EIVISON plus (kit-9903, MXB), DAB kit (ZL1-9019, ZSGB-BIO) per the manufacturers protocol were used for coloration. Finally, the tumor sections were stained with hematoxylin. All stained slides were scanned under high magnification $(\times 20$ objective lens), and four non-overlapping areas (excluding necrotic areas) were selected from each tumor tissue, which was rich in $\mathrm{FoxP}^{+}$(ab20034, Abcam) or $\mathrm{CD}^{+}$(ab9328, Abcam) TILs. The final number of TILs for each section was based on the average of the four fields. The number of TILs was counted using Image J software (http://rsb. info.nih.gov/ij/).

\section{Evaluation of the percent of PD-L1 ${ }^{+}$blood vessels and the positive rate of VEGFA and HIF-1a in tumor}

For evaluation of the percent of CD $34^{+}$PD-L1 ${ }^{+}$vessels in tumor tissues. All patient sections were double-stained with anti-CD34 antibody (ZA-0550, ZSGB-BIO) and antiPD-L1 antibody (66248-1-lg, Proteintech) using a doublestaining kit (DS-0001, ZSGB-BIO). First, the mostabundant sites of blood vessels were selected under fourfold magnification of microscope, and four sites were randomly selected under a 40-fold objective lens, and the proportion of $\mathrm{PD}-\mathrm{L}^{+}{ }^{+} \mathrm{CD} 34^{+}$cells in all $\mathrm{CD} 34^{+}$vessels was calculated and averaged. The average percentage was used as the final result for each section. To evaluate the positive expression of VEGFA (ab1316, Abcam) and hypoxia-inducible factor $\alpha$ (HIF-1 $\alpha)$ (ab113642, Abcam), stained sections were digitally analyzed at $\times 400$ resolution using an Olympus BX-UCB. HIF- $1 \alpha$ and VEGFA expression were scored by the $\mathrm{H}$-score system, respectively. $H$ score calculation method is based on previous literature ${ }^{15}$.

\section{Flow cytometry}

Preparation of single-cell suspension of mouse tumor tissues by mechanical grinding. All single-cell suspensions were incubated with rat anti-mouse CD16/CD32 blocking antibody $(4 \mu \mathrm{g} / \mathrm{ml})$ for $15 \mathrm{~min}$ after thorough filtration and precipitation, stained with fluorescein-conjugated antibodies, multiple washed with PBS, and resuspended in 7AAD (exclude non-viable cells). For anti-mouse CD4 (Clone RM4-5, BD Biosciences), anti-mouse CD8 (clone 53-6.7, eBioscience), anti-mouse CD45 (clone 30-F11, Biolegend), anti-mouse CD25 (PC61.5, eBioscience), antimouse PD-L1 (clone 10 F.9G2, BioLegend) and antimouse CD31 (clone MEC 13.3, BD Biosciences) staining, after incubation for $1 \mathrm{~h}$, cells were washed with PBS for three times ( $1500 \mathrm{rpm}, 5 \mathrm{~min}$ each), then detected by flow cytometry (BD FACSCanto II). For FoxP3 staining, after incubation, cells were washed and fixed with $1 \mathrm{ml}$ of fixation \& permeabilization solution (BD Biosciences) for 30-60 min, and washed twice with Perm Wash (BD Biosciences). Intracellular staining with anti-FoxP3 antibody (clone FJK-16s, eBioscience) was performed for $1 \mathrm{~h}$. The next steps are the same as described above.

\section{Immunofluorescence}

Fresh frozen tumor sections (stored at $-80^{\circ} \mathrm{C}$ ) were fixed in precooled $4 \%$ paraformaldehyde for $15 \mathrm{~min}$ at room temperature. The fixed frozen samples were permeabilized with $0.2 \%$ Triton X-100 (Applichem) in PBS for 10 minutes. After washing with PBS for three times, 5 min per time, all samples were incubated with blocking solution containing 1\% BSA (Sigma), 0.01\% Triton X-100 and $10 \%$ FBS in PBS for $1 \mathrm{~h}$. Next, the sections were incubated with primary antibody at $4^{\circ} \mathrm{C}$ overnight. Primary antibodies used were as follows: FITC-CD31 (clone MEC 13.3, BioLegend), rabbit anti-mouse CD31 (ab28364, Abcam), APC-PD-L1 (clone 10 F.9G2, BioLegend), rabbit anti-mouse PD-L1 (LS-C746930, LifeSpan BioSciences), rat anti-mouse CD4 (Clone RM4-5, BD Biosciences), rat anti-mouse CD8 (clone 53-6.7, eBioscience) and rat anti-mouse FoxP3 (clone FJK-16s, eBioscience). Then, the sections were rewarmed at room temperature for $15 \mathrm{~min}$, followed by washing three times in PBS for $5 \mathrm{~min}$ per time. At last, tissue sections were stained with secondary antibodies and incubated for $1 \mathrm{~h}$ at room temperature. The secondary antibodies used included: donkey anti-goat AF488 (SA5-10086, Invitrogen); donkey anti-rat AF488 (A-21208, Invitrogen); donkey anti-goat AF568 (A-11057, Invitrogen); donkey antirabbit AF647 (A-31573, Invitrogen); washed three times again in PBS, and subsequently stained with antifluorescence quencher (including DAPI). All stained sections were stored at $-20^{\circ} \mathrm{C}$ and used for image acquisition using Zeiss Imaginer-Z2. According to previous study ${ }^{10}$, if $\mathrm{CD}_{4}^{+}, \mathrm{CD}^{+}$, or $\mathrm{FoxP}^{+} \mathrm{T}$ cells are contained within a $25-\mu \mathrm{m}$ radius from the $\mathrm{CD} 31^{+}$vascular structure, it is defined as "perivascular immune cells".

\section{In vivo experiment}

C57BL/6 J female mice (6 weeks) were purchased from the Model Animal Center of Nanjing University. All experimental procedures were in accordance with the protocols approved by the Institutional Animal Care and Research Advisory Committee of Tianjin Medical University. In order to construct tumor-bearing mouse models of B16 or MC38 cells, $1 \times 10^{6} / 100 \mu \mathrm{l}$ cancer cells were injected subcutaneously into each mouse. B16 and MC38 tumors were grown for several weeks and observed 
every other day. From the 11th day, mice were randomly divided into PBS, anti-VEGF (bevacizumab, $10 \mathrm{mg} / \mathrm{kg}$, every 3 days), anlotinib (1.5 mg/kg, every day) and combination groups, each group was given different treatment, intraperitoneal injection of anti-VEGF or gavage anlotinib. According to the previous study ${ }^{10}$, The calculation formula of tumor volume is $V=\pi \times$ width $\times$ width $\times$ length $/ 6$. We set the B16 tumors reached a mean volume of $2000 \mathrm{~mm}^{3}$ as the end time of mice' survival and set the MC38 tumors reached a mean volume of $1000 \mathrm{~mm}^{3}$ as the end time of mice' survival.

\section{T-cell depletion}

In T-cell depletion experiments, we used antibodies included CD4 depletion antibody (clone GK1.5, BioXcell) and CD8 depletion antibody (clone 53-6.7, BioXcell). For 1 day immediately before treatment with anlotinib, mice were separately treated i.p. twice weekly with $200 \mu \mathrm{g}$ of the CD8 depletion antibody, CD4 depletion antibody and combination of both in different groups. Depletion efficiency was determined by flow cytometric analysis of mice spleen tissue.

\section{bEnd.3 model}

Mouse endothelial cells of bEnd.3 were transduced with a lentiviral expression vector (overexpressed CD274 (PDL1)). In all, $1 \times 10^{6}$ empty vector or CD274-transduced bEnd. 3 cells were injected intratumorally (i.t.) into B16 tumors on day13, followed by an additional i.t. injection at next week. Treatments with anlotinib were carried out as described for the above experiments before 1 day injection with bEnd,3 cells.

\section{Statistics}

All Statistical analyses were performed using SPSS v.21 (IBM Corp), and all statistical graphs and survival curves were generated using GraphPad Prism 6 (USA, GraphPad Software). Patients' overall survival (OS) and progressionfree survival (PFS) were estimated by the Kaplan-Meier method and compared using the log rank's test. One-way analysis of variance was performed for tumor growth analysis. Differences between the two groups were calculated using an unpaired Student's $t$ test. $P<0.05$ was considered significant statistical differences.

\section{Results}

\section{The VECs in different human cancers express PD-L1}

Although it has been reported in the literature that PD-L1 is expressed on the VECs, the status of PD-L1 expression on VECs inside tumor tissues has not been detailed reported. We analyzed the PD-L1 expression in various samples of human cancers including lung adenocarcinoma $(n=35)$, colon cancer $(n=50)$, renal cancer $(n=32)$, and the corresponding para-carcinoma tissues. Here we found that PD-L1 expression in the normal lung tissue was low or none, whereas a significant percentage of $\mathrm{CD}^{+} 4^{+}$blood vessels exhibited high PD-L1 expression in lung adenocarcinoma tissue, colon cancer and renal cancer (Fig. 1a, b). Moreover, we observed that the high expression of PD-L1 on VECs predicted a poor PFS $(P=0.014)$ and OS $(P=0.021)$. As for the relationship between the expression of PD-L1 and the characteristics of patients with lung adenocarcinoma $(n=$ 106), we did not find a significant correlation between the percentage of $\mathrm{CD}_{3} 4^{+} \mathrm{PD}^{\mathrm{L}} 1^{+}$vessels and any clinical variables, even though $P$ value is closed to significant point (0.066) in T stage (Fig. 1c and Table S1). Though in previous studies $^{16,17}$, the tumor-expressing PD-L1 was always a poor prognostic factor for lung adenocarcinoma, we also did not find a close relation between the PD-L1 expressed in tumor (T-PD-L1) and VECs (VEC-PD-L1) $(r=0.118 p=0.227)$ (Fig. 1d). This result indicates that the expression of PD-L1 on VECs does not depend on the expression of PD-L1 on tumor cells, but affects independently the prognosis of lung adenocarcinoma.

\section{PD-L1 expressed on human VECs participates in immune regulation}

As a recognized co-inhibitory molecular for T cells, PD-L1 can bind to PD-1 to inhibit the biological activity of T lymphocytes, thereby inhibiting T-cell-mediated tumor-specific immunity. According to previous literature ${ }^{18,19}$, the immune microenvironment in tumor tissues is closely related to the balance of $\mathrm{CD}^{+}{ }^{+} \mathrm{T}$ cells and FoxP3 ${ }^{+}$Treg cells. In three vasculature-rich tumors (lung, colon, and kidney cancers), we found that VECs with overexpression of PD-L1 were concomitantly with higher infiltration of FoxP3 ${ }^{+}$Treg cells $(r=$ $0.348, P=0.026)$, but with lower infiltration of $\mathrm{CD}^{+} \mathrm{T}$ cells $(r=-0.398, P=0.010)$ in lung adenocarcinomas (Fig. 2a, b). In order to verify this result, we extended to additional tumors including specimens of colon and kidney cancer, and found that overexpression of PD-L1 on VECs was also associated with fewer $\mathrm{CD}^{+}{ }^{+} \mathrm{T}$ cells and more FoxP3 ${ }^{+}$Treg cells in these two cancer tissues (Fig. 2c-e). These findings indicate that the upregulation of PD-L1 expression on tumor VECs may inhibit the infiltration of $\mathrm{CD}^{+} \mathrm{T}$ cells and activate FoxP3 ${ }^{+}$Treg cells.

\section{PD-L1 expression on VECs affects the proliferation and activation of immune cells}

Monocytes were extracted from the peripheral blood of healthy volunteers with complete consent, activated in vitro with anti-CD3 and IL-2 for $72 \mathrm{~h}$, and co-cultured with VECs treated by the supernatant of A549 cells for $48 \mathrm{~h}$ (Fig. 3a). We found that CM of tumor could significantly increase PD-L1 expression on VECs at $48 \mathrm{~h}$ (Fig. $3 \mathrm{~b})$. In addition, PD-L1 expression on VECs could be inhibited by treatment with anlotinib (Fig. 3c). After VECPD-L1 inhibition (treated with Anlotinb or anti-PD-L1 monoclonal antibody), we observed an enhancement in 


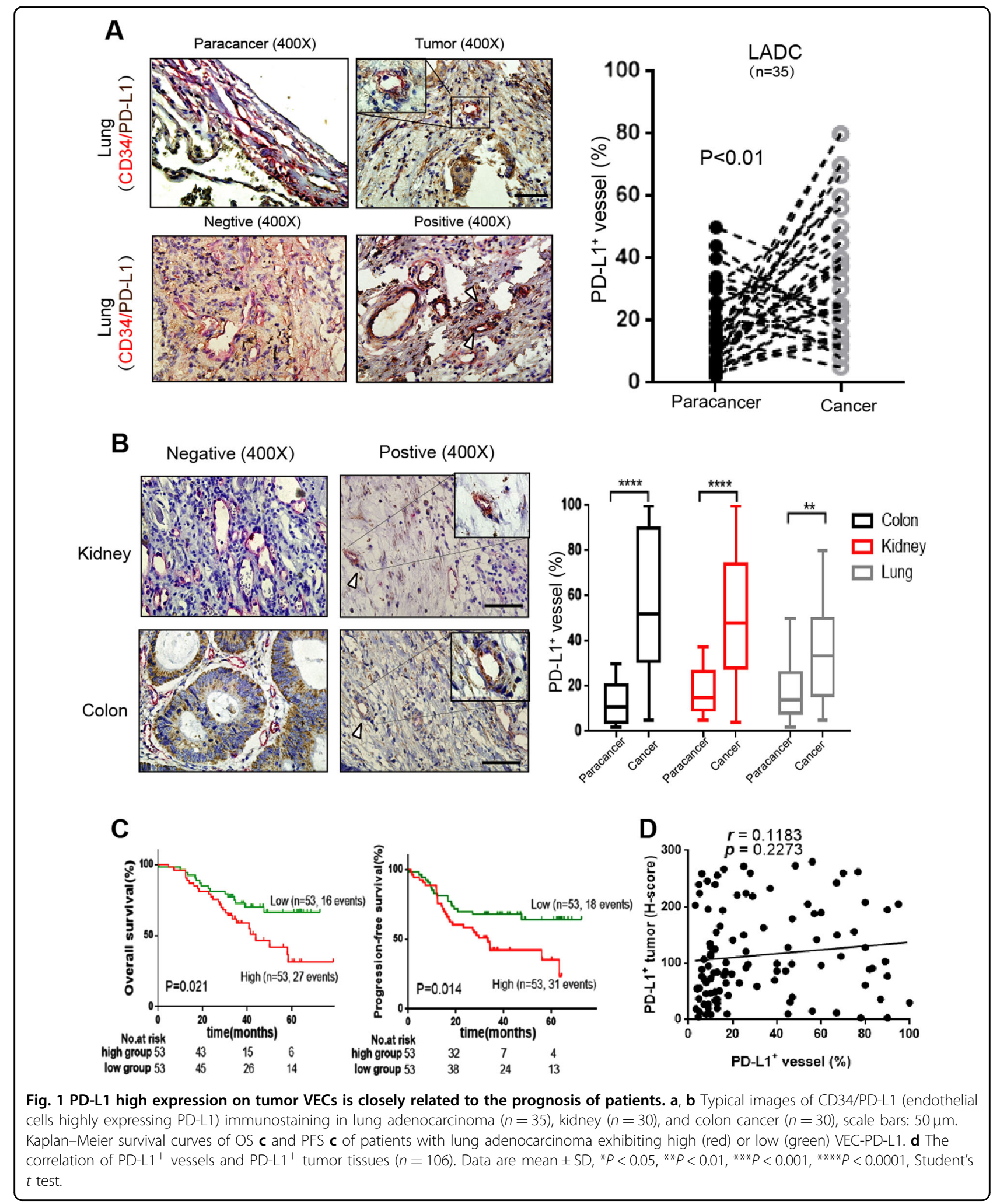

the proliferation capacity of $\mathrm{CD}^{+} \mathrm{T}$ cells and the activation of $\mathrm{CD}^{+} \mathrm{T}$ cells (increased secretion of IFN- $\gamma$ and granzyme B) (Fig. 3d, e). Furthermore, anlotinib or
anti-PD-L1 drug could effectively decrease the proliferation of FoxP3 ${ }^{+} \mathrm{T}$ cells (Fig. 3d, e). Through using a liquid chip to detect IL-4, IL-10, and TNF- $\alpha$ in the supernatant 

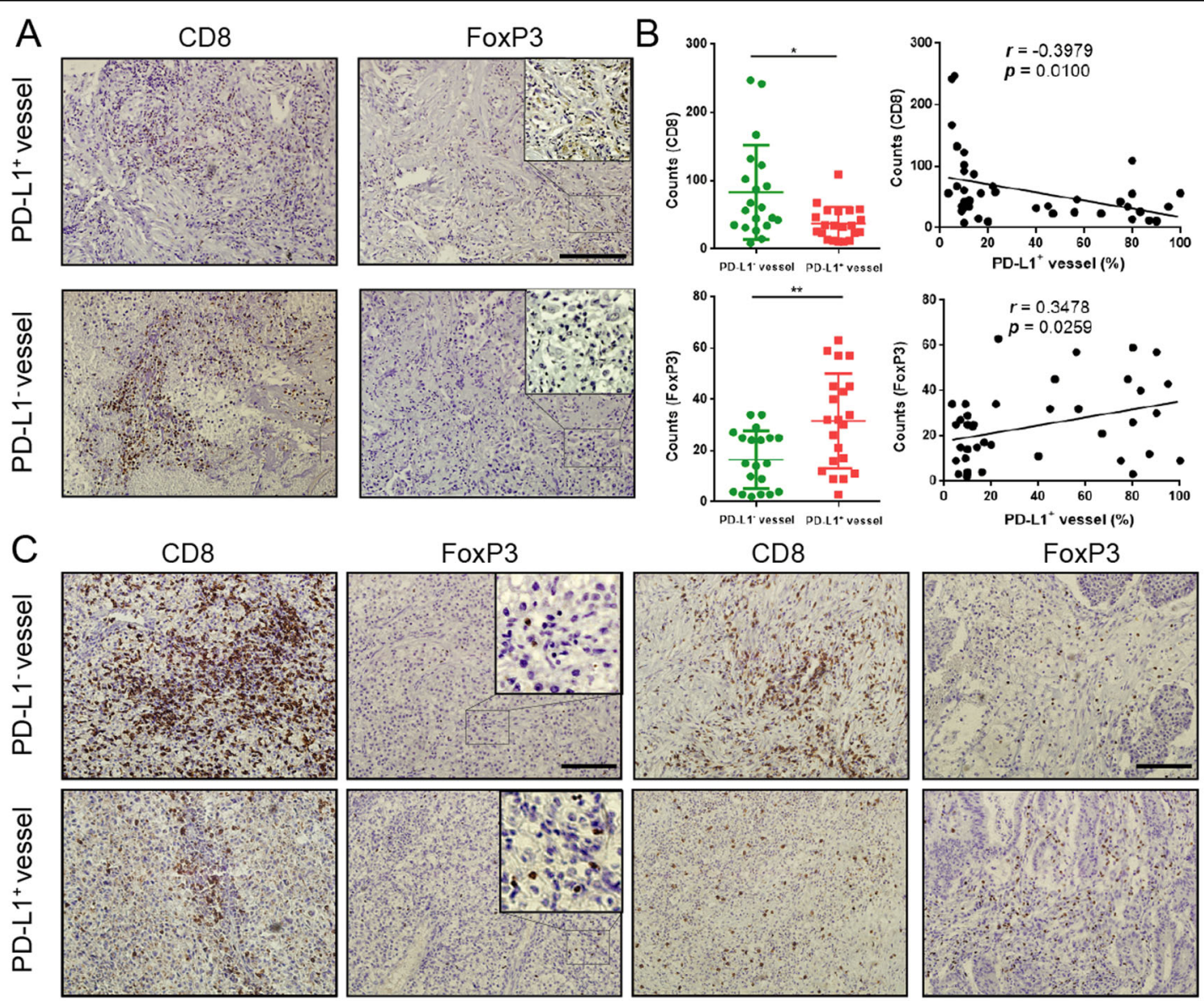

D

Kidney cancer (200X)

$\mathrm{E}$

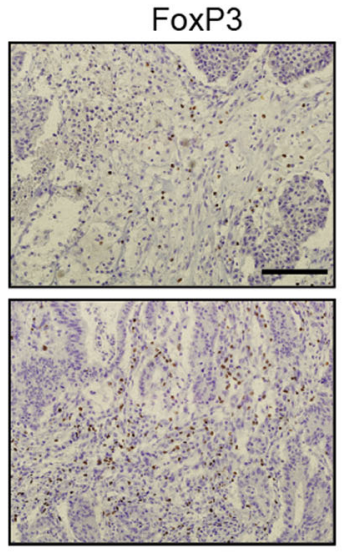

Colon cancer (200X)
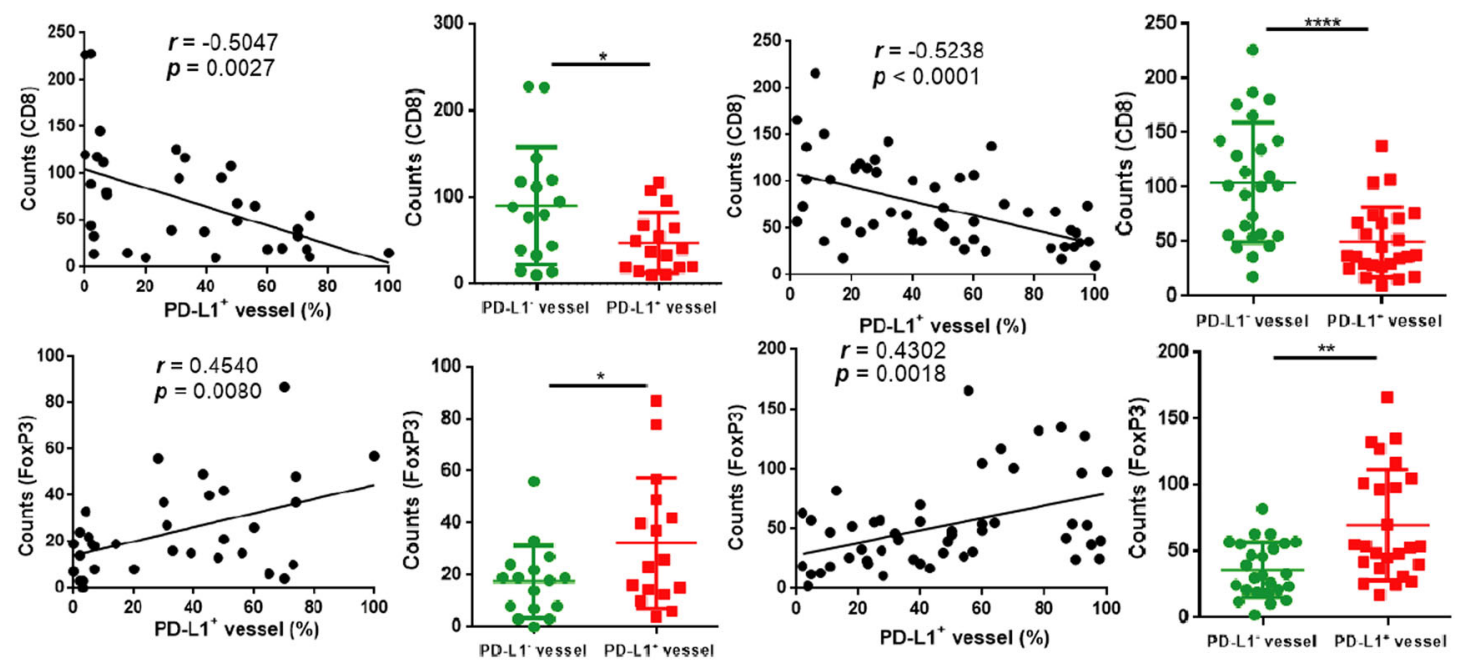

Kidney cancer

\section{Colon cancer}

Fig. 2 PD-L1 high expression on VECs is closely related to the infiltration of immune cells. a, c Representative images of CD8 $8^{+} T$ cells and FoxP3 $^{+}$T cells in sections taken from subjects with either PD-L1 ${ }^{+}$or PD-L1 ${ }^{-}$vessels in lung, kidney, and colon cancer. Original magnification, $\times 200$. The number of intratumoral CD8 ${ }^{+} T$ cells and FoxP3 ${ }^{+} T$ cells from patients' sections (kidney or colon cancer) associated with the percentage of PD-L $1^{+}$vessels. The determination as high and low were grouped by the median values. Lung adenocarcinoma $(n=41) \mathbf{b}$, colon cancer $(n=50)$ e and kidney cancer $(n=32) \mathbf{d}$; scale bars: $100 \mu \mathrm{m}$. Data are mean \pm SD. ${ }^{*} P<0.05,{ }^{*} P<0.01,{ }^{* * *} P<0.001,{ }^{* * *} P<0.0001$, Student's $t$ test. 


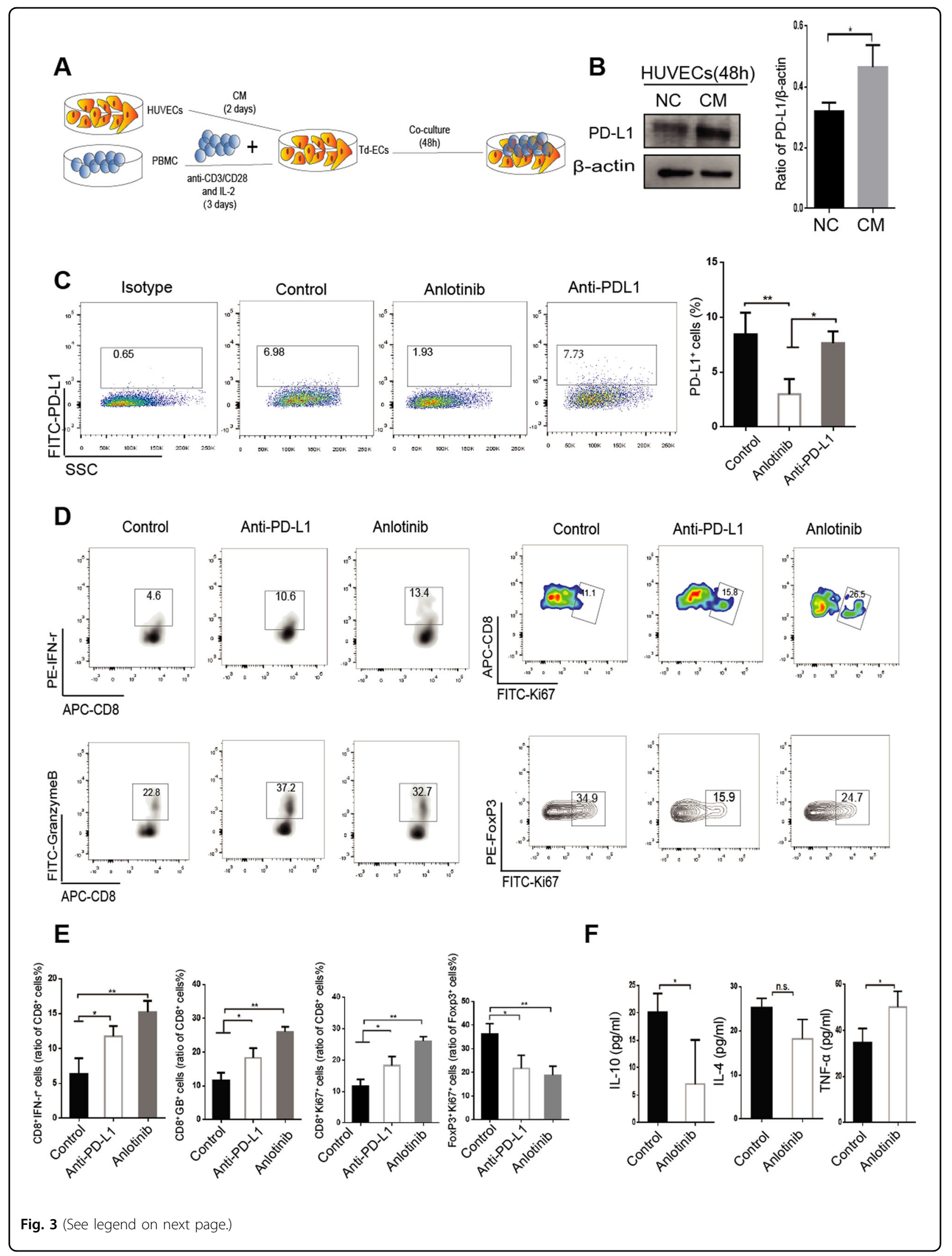


(see figure on previous page)

Fig. 3 PD-L1 expression on endothelial cells affects the proliferation and activation of immune cells. a PBMC was extracted from normal blood and treated with $2.5 \mathrm{\mu g} / \mathrm{ml}$ anti-CD3 antibody and IL-2 (100 U/ml) for $72 \mathrm{~h}$, and co-cultured with tumor-derived endothelial cells (Td-EC) for $48 \mathrm{~h}$. b HUVECs were treated with the supernatant of tumor cells (CM) for $48 \mathrm{~h}$. c PBMC and Td-EC were treated with control, anlotinib (0.1 $\mu \mathrm{m})$ or antiPD-L1 antibody $(4 \mathrm{\mu g} / \mathrm{ml})$. d Detection of the $\mathrm{CD}^{+} \mathrm{IFN}-\gamma^{+}, \mathrm{CD}^{+} \mathrm{Ki} 7^{+}, \mathrm{CD}^{+} \mathrm{GranzymeB}^{+}$, and FoxP3 ${ }^{+} \mathrm{T}$ cells by flow cytometry. e Quantification of the above positive cells in the immune cells. $\mathbf{f}$ Quantification of cytokine levels in co-culture supernatants (2 days) by liquid microarray. Data are mean \pm SD. ${ }^{*} P<0.05,{ }^{*} P<0.01,{ }^{* *} P<0.001$, Student's $t$ test.



of co-culture, we found that anlotinib significantly inhibited the secretion of IL-10 and IL-4 and promoted the secretion of TNF- $\alpha$ (Fig. 3f). These results suggest that VEC-PD-L1 can affect the proliferation and activation of $\mathrm{CD}^{+} \mathrm{T}$ cells, as well as Treg cells.

\section{Anlotinib inhibits PD-L1 expression upregulated by VEGFA and HIF-1a via AKT signals on VECs}

By staining paraffin sections of lung adenocarcinoma, we found that the VECs with PD-L1 overexpression simultaneously revealed higher expression of VEGFA, HIF- $1 \alpha$, and microvascular density compared with negative group (Fig. S1a, b). This result suggests that the number of blood vessels, angiogenic growth factors, and HIF expression in tumor tissues are probably closely related to VEC-PD-L1.

In agreement with the results of our experiment in vitro, PD-L1 expression on VECs was facilitated under conditions of CM of tumor, hypoxia or FBS, but this phenomenon was not observed only with VEGFA stimulation (Fig. S1c). It suggests that VEGFA may not be the initiator for the PD-L1 expression on VECs alone and multiple factors, probably including HIF- $1 \alpha$, could drive PD-L1 expression. It has been reported in one literature that antiangiogenic drugs can block pro-angiogenic factors and improve hypoxia in tumor to a certain extent ${ }^{20}$. As a novel small molecule tyrosinase inhibitor, anlotinib can effectively inhibit angiogenesis and improve the perfusion of tumor tissues.

Based on the results of western blot, we found that anlotinib inhibited PD-L1 expression on VECs in hypoxia or in $\mathrm{CM}$ of tumor (Fig. S1d). In addition, anltotinib inhibited the activation of the PI3K/AKT pathway (Fig. 4a). Therefore, we blocked the AKT pathway, then found the inhibition of PD-L1 expression on HUVECs (Fig. 4b), and the same event was observed in the MRMEC and 
bEnd.3 cells (Fig. 4c and e). Moreover, we found that PDL1 expression was increased after the addition of AKT activator (SC-79), whereas it was inhibited again after the addition of AKT inhibitor (LY294002) (Fig. 4d and f). It indicates that the AKT pathway is involved in the regulation of PD-L1 in endothelial cells. In order to further investigate the mechanism of anlotinib's effect on endothelial PD-L1 expression, we selected the VEGFR phosphorylation inhibitor SU5408 (SU), the PDGFR phosphorylation inhibitor CP-673451 (CP) and the FGFR phosphorylation inhibitor FIIN-2 (FI) to block a variety of pro-angiogenic receptors. We found that the blockage of phosphorylation of VEGFR, PDGFR, or FGFR could downregulate the PD-L1 expression on VECs via inhibiting the activation of the AKT pathway (Fig. S1e).

\section{Anlotinib inhibits PD-L1 expression on VECs, improves the immune microenvironment via increasing the ratio of CD8/ FoxP3 and suppresses tumor growth}

To evaluate the impact of tumor-endothelial PD-L1 on the infiltration of $\mathrm{CD}^{+} \mathrm{T}$ cells and $\mathrm{FoxP}^{+} \mathrm{T}$ cells, we selected two xenograft animal models (MC38 and B16 cell lines). According to previous studies ${ }^{21,22}$, inhibition of PD-L1 expression in stromal cells is essential for antitumor immunity of B16 melanoma. On the contrary, the PD-L1 expression in MC38 colorectal adenocarcinoma cells is sufficient to suppress antitumor immunity. Therefore, B16 xenograft model was selected as the observation to better analyze the impact of PD-L1 expression on tumor VECs.

According to previous study ${ }^{10}$, anti-VEGF therapy upregulates PD-L1 expression on VECs in response to increased IFN- $\gamma$ secreted by the perivascular $\mathrm{CD}^{+}$ $\mathrm{T}$ cells. In our study, we found the similar phenomenon that the blockade of VEGF (bevacizumab) promoted VEC-PD-L1 in vitro experiment (Fig. S1f). In addition, anlotinib inhibited endothelial PD-L1 expression induced by bevacizumab (Fig. S1f). Therefore, we selected bevacizumab as a positive control group to observe the endothelial PD-L1 expression and the infiltration of immune cells in tumor tissues when angiogenesis was inhibited. In addition, we found that both bevacizumab and anlotinib had the similar angiogenesis inhibitory effects (Fig. S2A).

In the B16 xenograft model, we found that anlotinib reduced the mortality in mice and decreased the volume and weight of tumors (Fig. 5a-d). In the MC38 group, anlotinib also decreased the size of the transplanted tumors (Fig. S2b). However, treatment with bevacizumab increased the mortality, tumor volume, and tumor weight of mice. When combined with anlotinib during bevacizumab treatment, it significantly decreased the mortality and tumor volume of mice compared with the bevacizumab alone group (Fig. 5a-d). Based on the results in vitro experiments, we speculated that the different result of treatment might be closely related to endothelial PD-L1 expression. Therefore, we found that anlotinib and combined group significantly inhibited VEC-PD-L1, whereas bevacizumab strongly upregulated VEC-PD-L1 in tumor-bearing mice (Fig. $5 \mathrm{e}-\mathrm{g}$ ). In order to further study the status of PD-L1 expression on tumor cells, we used flow cytometry and immunofluorescence (IF) staining and found that neither anlotinib, bevacizumab or the combination of them had any significant effects on PD-L1 expression in non-VECs in tumor tissues, nor on PD-L1 expression in tumor cells (Fig. $5 \mathrm{f}$ and Fig. S2c). These results again suggest that VEC-PD-L1 is closely related to the immune microenvironment of tumor tissues and the efficacy of antiangiogenesis therapy.

In further analysis of the infiltration of immune cells, we found that compared with the control group, anlotinib and combination treatment group significantly inhibited the aggregation of $\mathrm{CD} 45^{+} \mathrm{CD} 4{ }^{+} \mathrm{CD} 25^{+} \mathrm{FoxP}^{+} \mathrm{T}$ cells in the tumor tissues (Fig. 6a, b). However, treatment with bevacizumab did not inhibit or even slightly increase the infiltration of $\mathrm{FoxP}^{+} \mathrm{T}$ cells break through in the $\mathrm{B} 16$ xenograft model, and had a lower the ratio of CD8/FoxP3 compared with other groups (Fig. 6a, b). However, anlotinib group had a significant higher the ratio of CD8/ FoxP3 (Fig. 6c). In the MC38 xenograft model, we found the similar phenomenon that anlotinib promoted the ratio of CD8/FoxP3 compared with the control group (Fig. 6c).

In addition, IF was used to analyze the degree of infiltration of immune cells in tumor tissues, and we found that there was no significant difference in the infiltration number of $\mathrm{CD}^{+} \mathrm{T}$ cells between different groups (Fig. $6 \mathrm{~d}, \mathrm{e})$. Although we did not find a significant increase in the infiltration number of $\mathrm{CD}^{+} \mathrm{T}$ cells in anlotinib group, there was a conspicuous upward trend compared with the control group (Fig. 6d, e). Moreover, it is considered that the $\mathrm{CD}^{+} \mathrm{T}$ cells around blood vessels as "inefficacious" immune cells can not to penetrate the immune barrier and infiltrate into tumor tissue. By counting we found a large amount of $\mathrm{CD}^{+} \mathrm{T}$ cells clustered around the blood vessels in the bevacizumab group, while infiltrated $\mathrm{CD}^{+} \mathrm{T}$ cells were much lower than those of the anlotinib or combination treatment group (Fig. 6f). In addition, we found the infiltration number of $\mathrm{FoxP}^{+} \mathrm{T}$ cells was significantly reduced in anlotinib group, thereby improving the ratio of $\mathrm{CD} 8$ / FoxP3 (Fig. 6e-g). On the other hand, bevacizumab group had a lowest ratio of CD8/FoxP3 compared with the other groups, which exacerbated the immunosuppressive microenvironment (Fig. 6f, g). In addition, in the MC38 xenograft model, we observed that anlotinib group could also significantly improve the ratio of CD8/FoxP3 (Fig. $6 \mathrm{~g}$ ). These results suggest that anlotinib can break through the immunosuppressive barrier of VEC-PD-L1 


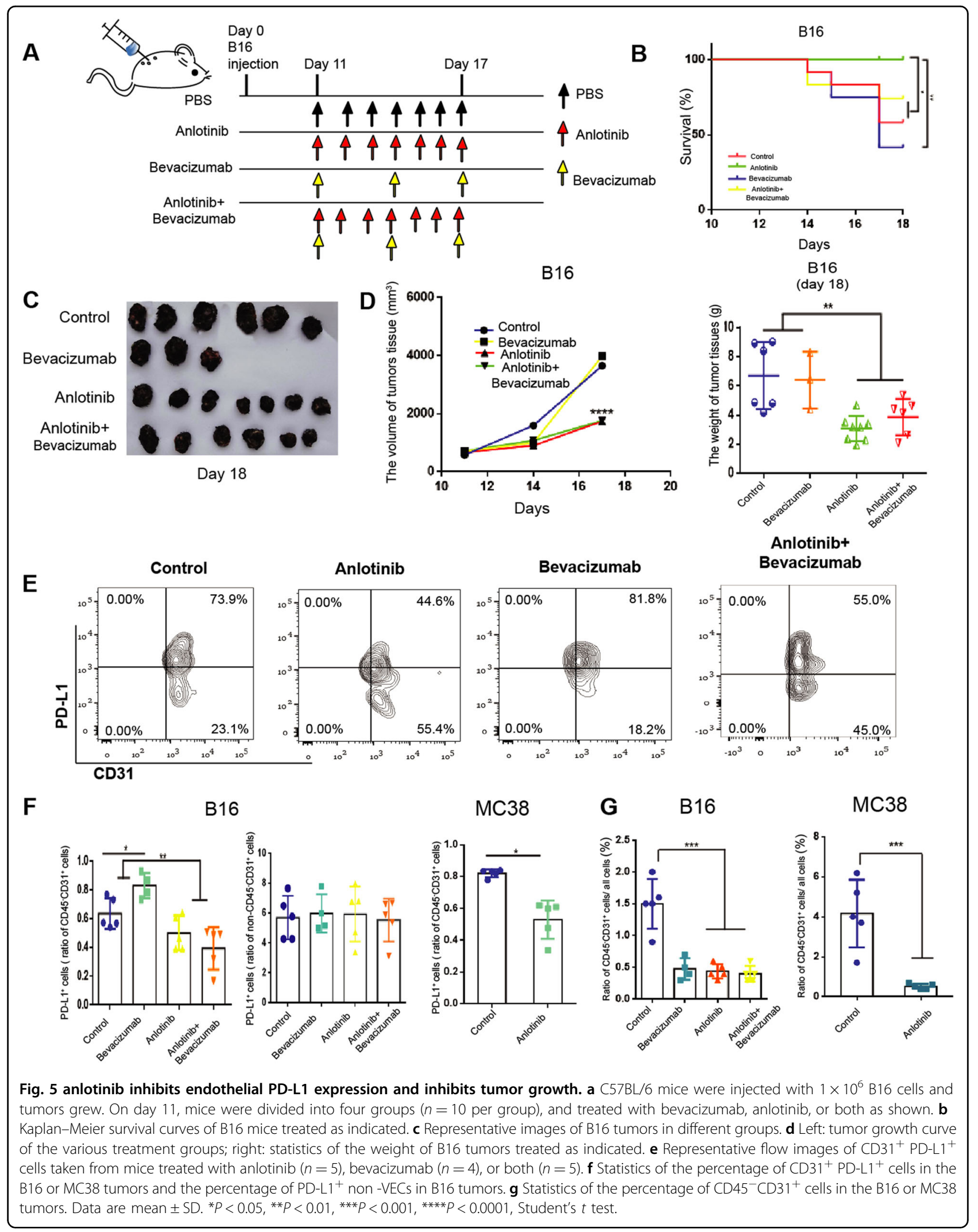







(see figure on previous page)

Fig. 6 anlotinib improves the immune microenvironment via increasing the ratio of CD8/FoxP3. a Representative flow images of CD4 ${ }^{+}$ $\mathrm{CD}_{25}{ }^{+} \mathrm{FoxP3}^{+} \mathrm{T}$ cells taken from B16 tumors treated as above indicated. $\mathbf{b}, \mathbf{c}$ Statistics of the percentage of CD4 $4^{+} \mathrm{T}$ cells, $\mathrm{CD} 8^{+} \mathrm{T}$ cells, FoxP3 ${ }^{+} \mathrm{T}$ cells and ratio of CD8/FoxP3 in the B16 or MC38 tumors. d Representative images of CD31 immunostaining (red), 4',6-diamidino-2-phenylindole (DAPI) nuclear staining (blue) and markers of immune cells (CD4, CD8, and FoxP3) (green) of B16 tumors ( $n=5$ per group, except for Bev group $n=4)$ treated as above indicated. Scale bars, $100 \mathrm{~mm}$. e Quantification of CD4 ${ }^{+} \mathrm{T}$ cells, CD8 ${ }^{+} \mathrm{T}$ cells, FoxP3 ${ }^{+} \mathrm{T}$ cells in B16 tumors. f Statistics of perivascular and without perivascular CD8 ${ }^{+} T$ cells in the B16 tumors. $\mathbf{g}$ Statistics of ratio of CD8/FoxP3 in the B16 or MC38 tumors. All data are exhibited as mean \pm SD. Statistical differences were assessed using the unpaired Student's test. ${ }^{*} P<0.05,{ }^{* *} P<0.01,{ }^{* *} P<0.001,{ }^{* * *} P<0.0001$.

and significantly increase the ratio of CD8/FoxP3 in tumor tissues.

PD-L1 expressed on VECs affects the efficacy of anlotinib

To determine the effect of immune function of anlotinib treatment, we used CD4 and CD8 depletion antibodies to deplete $\mathrm{CD} 4^{+} \mathrm{T}$ and $\mathrm{CD} 8^{+} \mathrm{T}$ cells. We found that the efficacy of anlotinib was significantly reduced after depletion of $\mathrm{CD}^{+} \mathrm{T}$ cells, whereas depletion of $\mathrm{CD} 4^{+}$ $\mathrm{T}$ cells alone had no significant effect on anlotinib's efficacy (Fig. S3a-c). These results indicate that the function of $\mathrm{CD}^{+} \mathrm{T}$ cells has an important role in treatment with anlotinib.

To further determine the effect of PD-L1 expression on VECs, CD274 (PD-L1)-overexpressing lentivirus vector was transduced to bEnd. 3 cells to construct a CD274bEnd.3 $(+)$ cell line, which could stably overexpressing PD-L1. We injected bEnd.3 (-) or CD274-bEnd.3 (+) cells into tumor tissues separately, and found that the tumor tissues with bEnd.3 $(+)$ group (injected intratumorally CD274-bEnd.3 $(+)$ ) had a faster growth rate compared to the bEnd.3 (-) group (injected intratumorally bEnd.3 (-)) (Fig. $7 \mathrm{a}-\mathrm{c}$ ). In addition, in order to confirm whether VEC-PD-L1 had a role on the efficacy of anlotinib, we injected bEnd.3 (-) or CD274-bEnd.3 (+) cells intratumorally into tumor tissues separately with anlotinib treatment. Interestingly, we found that the therapeutic effect of anlotinib was assuaged in the anlotinib+bEnd.3 (+) group, and the B16 tumor tissues almost had the similar growth rate with the bEnd.3 (-) group. What's more, anlotinib showed a promising curative effect in the anlotinib+bEnd.3 (-) group compared with the bEnd.3 (-) group, both the volume and the weight of tumor tissues were significantly decreased (Fig. $7 \mathrm{a}-\mathrm{c})$.

To further analyze whether this immunosuppressive phenomenon was caused by VEC-PD-L1, we collected tumor tissues from mice for flow cytometry. We found that the number of $\mathrm{FoxP}^{+} \mathrm{T}$ cells was increased in bEnd.3 (+) group, whereas the ratio of CD8/FoxP3 was lower compared with bEnd.3 (-) group (Fig.7d, e). The number of FoxP3 ${ }^{+} \mathrm{T}$ cells was decreased with anlotinib treatment (Fig. 7d, e). Although there was no significant difference in the number of $\mathrm{CD} 8^{+} \mathrm{T}$ cells among different groups, we found the activity of $\mathrm{CD}^{+} \mathrm{T}$ cells was enhanced and the number of $\mathrm{CD}^{+} \mathrm{IFN}^{+} \gamma^{+}$cells was increased after treatment with anlotinib (Fig. 7e). In addition, our study has confirmed that CD274-bEnd.3 cells can promote the infiltration of $\mathrm{FoxP}^{+} \mathrm{T}$ cells, reduce the ratio of $\mathrm{CD} 8 / \mathrm{FoxP} 3$, and inhibit the function of $\mathrm{CD}^{+} \mathrm{T}$ cells with anlotinib treatment (Fig. 7d, e). These findings indicate that VEC-PD-L1 can not only affect the immune balance in tumor tissues, but also play an important regulatory role in the therapeutic effect of anlotinib.

\section{Discussion}

VECs have been considered as a source of nutrition for tumor. However, with the development of research, it has been found that abnormal vasculature in tumor microenvironment also combines with the abnormal pericytes, forming dysfunctional tumor blood vessels, which contribute to the formation of an immunosuppressive environment ${ }^{1}$. Some studies ${ }^{23,24}$ have confirmed the view that blood vessels are not only a nutrient supply channel in tumor microenvironment, but also one of the important roles in immune regulation. Several studies ${ }^{7,25,26}$ suggest that VECs expressing PD-L1 is involved in autoimmune disease such as ARDS in the lung or skin inflammation, etc., and is closely related to the development of Treg cells and the inactivation of $\mathrm{CD}^{+} \mathrm{T}$ cells. Most studies ${ }^{7,8,26}$ focus on the impact of the PD-L1 expression on tumor cells or autoimmune disease, but few focus on the status of PD-L1 expression on VECs and its role in the tumor microenvironment.

In this regard, our study has demonstrated that angiogenic growth factors (VEGFA) and hypoxia are closely related to the PD-L1 expression on VECs in tumor. This result suggests that abnormal tumor environment may promote the expression of PD-L1 on VECs. According to previous literature, the PD-L1 expression on stromal cells is closely related to clinical stage $\mathrm{e}^{27}$. We also found that the PD-L1 expression on VECs was closely related to clinical stage. However, owing to both the clinical stage and whole stromal cells were only rough index, wherein we may not find the real role of VEC-PD-L1, we further analyzed the correlation between PD-L1 expression on VECs and TNM stage (T stage, $\mathrm{N}$ stage, and $\mathrm{M}$ stage). We did not 
A



B

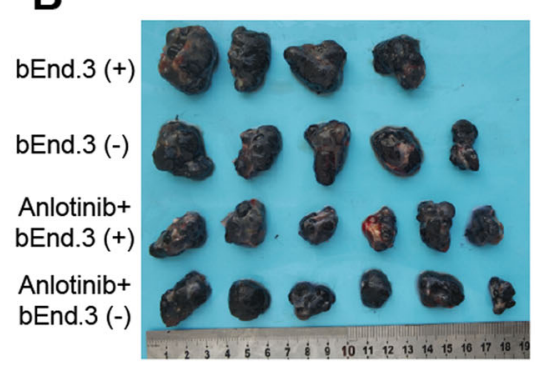

C $\left.{ }^{4000}\right] \rightarrow$ EEnd.3(-)
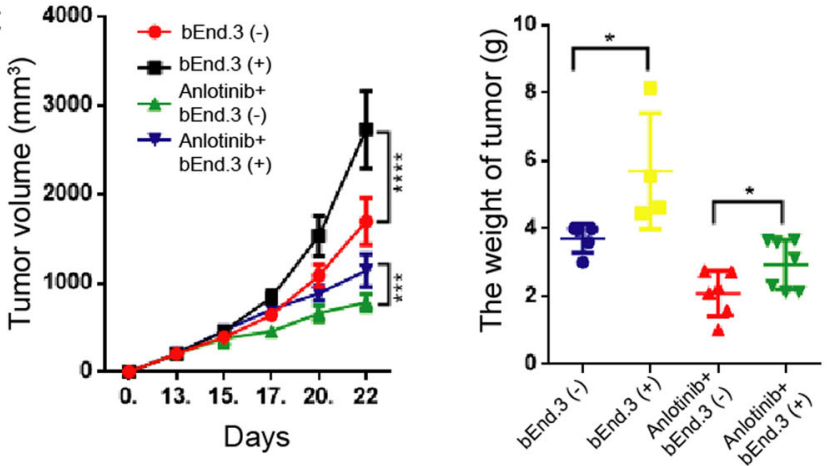

D b.End3 (-)

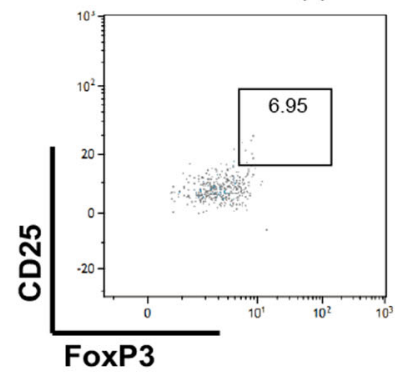

E

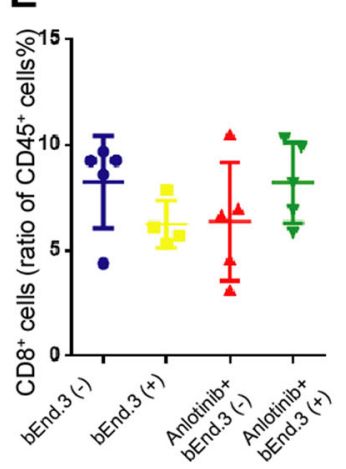

b.End3 (+)
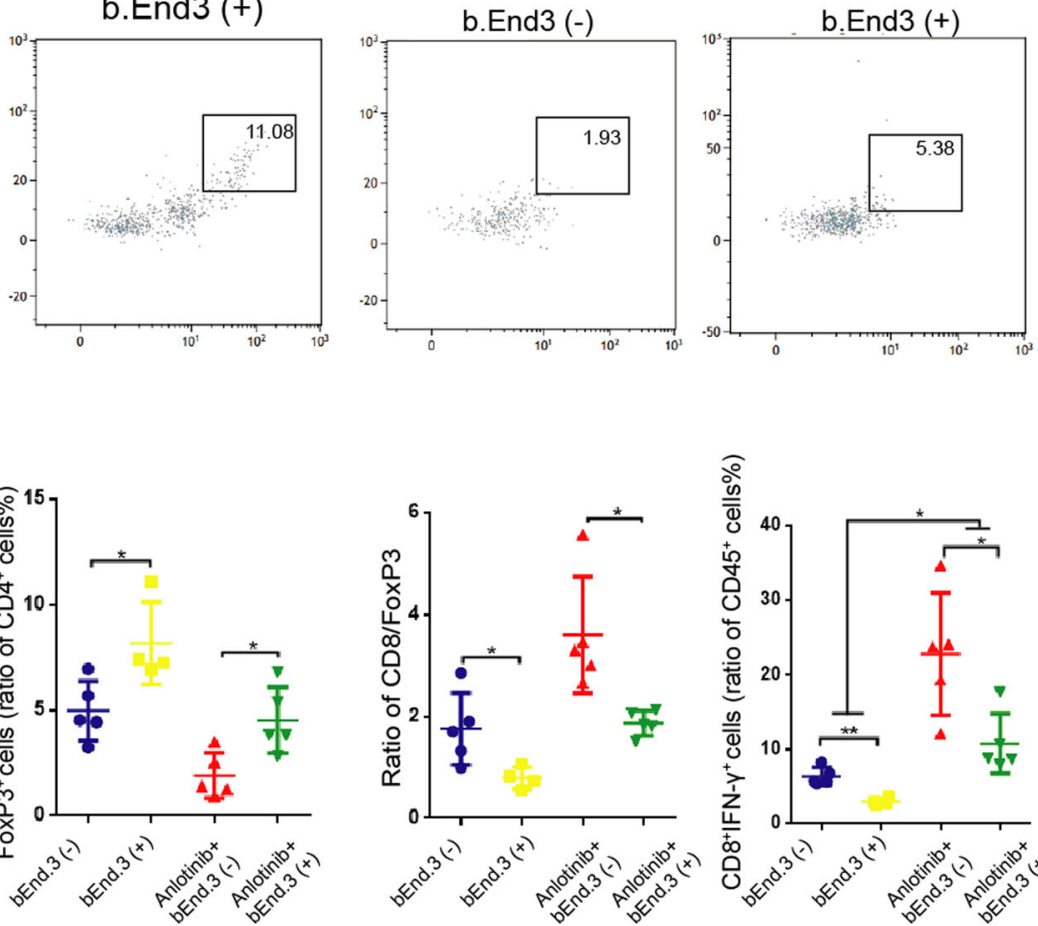
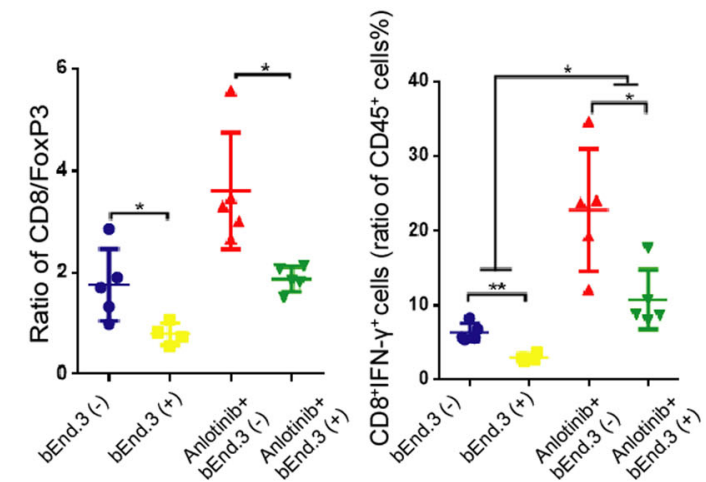

Fig. 7 (See legend on next page.) 
(see figure on previous page)

Fig. 7 Endothelial PD-L1 expression affects the efficacy of anlotinib. a C57BL/6 mice were injected with $1 \times 10^{6}$ B16 cells and tumors grew. On day 12, mice were divided into four groups ( $n=8$ per group), and treated with bEnd.3-vector, bEnd.3-CD274, PBS or anlotinib as shown. b Representative images of B16 tumor tissues treated as above indicated. c Left: tumor growth curve of the various treatment groups; right: Statistics of the weight of B16 tumors treated as indicated. $\mathbf{d}$ Representative flow images of $\mathrm{CD}^{+} \mathrm{CD}^{2} 5^{+} \mathrm{FoxP}^{+} \mathrm{T}$ cells taken from B16 tumors treated as indicated. e Statistics of the percentage of $\mathrm{CD}^{+}{ }^{+} \mathrm{T}$ cells, $\mathrm{CD} 8^{+} \mathrm{IFN}-\gamma^{+} \mathrm{T}$ cells, FoxP3 ${ }^{+} \mathrm{T}$ cells and ratio of CD8/FoxP3 in the tumors. Data are mean \pm SD. ${ }^{*} P<0.05,{ }^{* *} P<0.01,{ }^{* * *} P<0.001,{ }^{* * *} P<0.0001$.

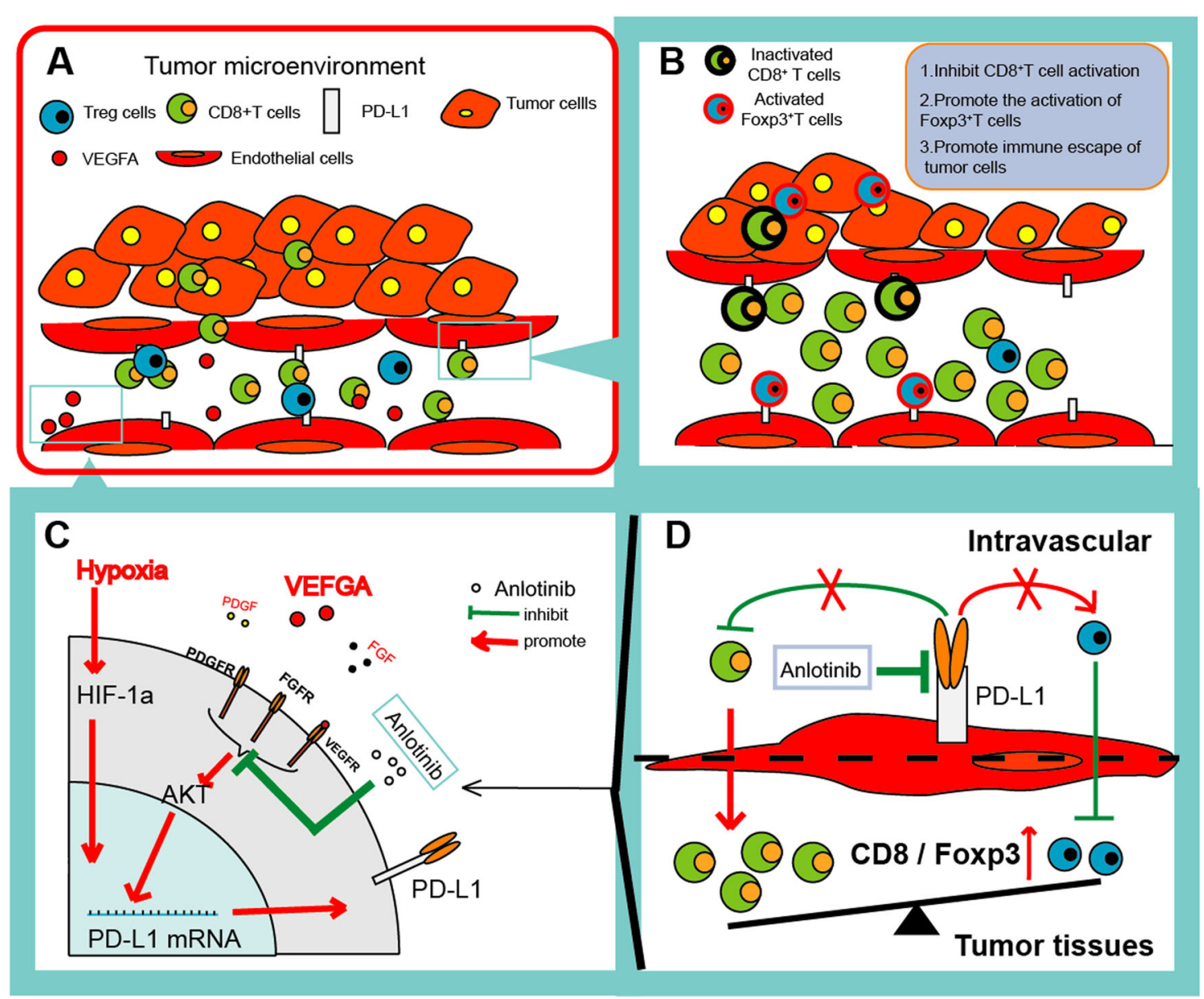

Fig. 8 A schematic representation of effects of PD-L1 expression on VECs in tumor microenvironment. a Pattern diagram of tumor immune microenvironment. $\mathbf{b}$ PD-L1 highly expressed on VECs in tumor tissues inhibits activation of CD8 ${ }^{+} \mathrm{T}$ cells and promotes immune escape of tumors. c anlotinib inhibits the expression of PD-L1 on VECs through the AKT pathway. $\mathbf{d}$ anlotinib improved the ratio of CD8/FoxP3 and broke the immune barrier by inhibiting the VEC-PD-L1.

find a significant relationship between high percentage of $\mathrm{CD}_{4} 4^{+} \mathrm{PD}-\mathrm{L}^{+}{ }^{+}$vessels and any clinical variables, but $P$ value was closed to significant point $(0.066)$ in $T$ stage. This result suggests that PD-L1 expression on VECs may be related to the $\mathrm{T}$ stage in primary tumor rather than $\mathrm{N}$ or $M$ stage. In addition, our study also found that the high expression of PD-L1 on VECs independently predicted a poor prognosis, but had no association with the PD-L1 expression on tumor cells. This observation suggests that PD-L1 expression on VECs may be a new prognostic factor in several tumors independently from the PD-L1 expression on tumor cells.

In our study, we found that bevacizumab promoted the PD-L1 expression on VECs. Based on the previous literatures $^{28,29}$ and our previous research ${ }^{30}$, we considered that bevacizumab could activate a variety of alternative pathways, such as the FGFR- and TGF- $\beta$-signaling pathway when it effectively neutralizes VEGFA, thereby activating the AKT pathway and finally causing upregulation of PD-L1 expression on VECs. In our vitro experiment, 
we found that blocking a variety of pro-angiogenic receptors (such as PDGFR, VEGFR2, and FGFR) by anlotinib inhibited the phosphorylation of AKT and inhibited PD-L1 expression on VECs induced by bevacizumab. Probably owing to the above effect, we found that anlotinib reduced the increase of mortality, tumor volume, and weight in mice induced by bevacizumab; simultaneously, PD-L1 was highly expressed on VECs in bevacizumab group, whereas inhibited on VECs in anlotinib group. These results suggest that VEC-PD-L1 is closely related to the immune microenvironment of tumor tissues and the efficacy of antiangiogenesis therapy.

According to previous studies ${ }^{4,18}$, the ratio of CD8/ FoxP3 is considered to be one of the markers of tumor immune microenvironment, and patients with a high ratio of CD8/FoxP3 always have a better prognosis. In this study, we found the PD-L1 expression on VECs also acted as an "immune barrier" to inhibit the infiltration of effector immune cells and affect the ratio of CD8/FoxP3.

$\mathrm{CD}^{+} \mathrm{T}$ cells around blood vessels are considered to be the immune cells that have not penetrated the immune barrier and infiltrated into tumor tissues. In order to evaluate the tumor microenvironment more realistically, the ratio of infiltration of $\mathrm{CD}^{+} \mathrm{T}$ cells (excluding the perivascular $\mathrm{CD}^{+} \mathrm{T}$ cells) and the $\mathrm{FoxP}^{+} \mathrm{T}$ cells was deeply analyzed. We discovered that anlotinib could successfully promote $\mathrm{CD}^{+} \mathrm{T}$ cells to break through the "immune barrier" of VEC-PD-L1, infiltrate tumor tissues and significantly increase the ratio of CD8/FoxP3 compared with control group. The occurrence of this phenomenon may be attributed to the downregulation of PDL1 expression on VECs, which leads to more easily infiltration of $\mathrm{CD}^{+} \mathrm{T}$ cells into tumor and the activation of those cells before they infiltrated into tumor tissues. Of course, the really regulation mechanism still need to be future studied. Whatever it is or not, our data indicate that PD-L1 expression on VECs may have an important role in antiangiogenic therapy combined with immunotherapy. These results also suggest that anlotinib can effectively reverse the immunosuppression phenomenon by inhibiting PD-L1 expression on VECs induced by single-target antiangiogenic drug.

In T-cell depletion experiments, we found that the efficacy of anlotinib was closely related to $\mathrm{CD}^{+} \mathrm{T}$ cells in tumor tissues. In addition, we found that when the endothelial cells overexpressing PD-L1 were injected into tumor tissues, the tumor immunosuppressive environment was further aggravated and the proportion of CD8/FoxP3 was declined. These results indicate that the PD-L1 expression on VECs can affect the growth of tumor tissues through the formation of immunosuppressive microenvironment. The efficacy of anlotinib was also inhibited after injection of bEnd.3 (+) cells, which reduced the ratio of CD8/FoxP3.
These results again suggest that the efficacy of anlotinib is closely related to the PD-L1 expression on VECs.

On the basis of these findings, a schematic representation for the effects of PD-L1 expression on VECs in tumor microenvironment was proposed (Fig. 8). Taken together, our findings indicate that the PD-L1 expression on VECs can realistically inhibit the immune-activation in microenvironment through promoting the aggregation of FoxP3 ${ }^{+} \mathrm{T}$ cells and inhibiting the function and infiltration of $\mathrm{CD}^{+} \mathrm{T}$ cells in tumor. Our study also indicates that anlotinib can regulate tumor immune microenvironment by inhibiting the expression of PD-L1 on VECs via AKT signaling pathway, which was not depend on its antiangiogenesis effects.

In summary, our experimental data demonstrate that VEC-PD-L1 has an important immunosuppressive role in the immune microenvironment and antiangiogenesis therapy, and illustrate the underlying mechanism of how anlotinib improved tumor immune microenvironment. The present study also provides a theoretical and experimental evidence for the combination of clinical anlotinib with immunotherapy.


and Technology Commission Projects (12ZCDZSY15600 to Kai Li), CSCO (Chinese Society of Clinical Oncology) Special Foundation for Tumor antiangiogenesis Therapy (Y-X2011-001 to Kai Li), National Natural Science Foundation of China (81802296 to Tingting Qin), Natural Science Foundation of Tianjin (18JCQNJC82500 to Tingting Qin), The Science of Technology Development Fund of Tianjin Education Commission for Higher Education (2017KJ201 to Tingting Qin) and CSCO (Chinese Society of Clinical Oncology) Special Foundation for Tumor antiangiogenesis Therapy (Y-S2014-011 to Jing Wang).

\section{Author details}

${ }^{1}$ Tianjin Medical University Cancer Institute and Hospital, National Clinical Research Center for Cancer, Tianjin, China. ${ }^{2}$ Key Laboratory of Cancer Prevention and Therapy, Tianjin, China. ${ }^{3}$ Tianjin's Clinical Research Center for Cancer, Tianjin, China. ${ }^{4}$ Department of Thoracic Oncology, Tianjin Lung Cancer Center, Tianjin Cancer Institute \& Hospital, Tianjin Medical University, Tianjin 300060, China

\section{Author contributions}

SL performed in vitro cells study and wrote the manuscript; TQ and YF performed in vivo animal experiments; ZL, YJ, YG, and JW contributed the evaluation of the data; $\mathrm{KL}$ revised the manuscript.

Conflict of interest

The authors declare that they have no conflict of interest.

\section{Publisher's note}

Springer Nature remains neutral with regard to jurisdictional claims in published maps and institutional affiliations.

Supplementary Information accompanies this paper at (https://doi.org/ 10.1038/s41419-020-2511-3).

Received: 12 February 2020 Revised: 16 April 2020 Accepted: 17 April 2020 Published online: 04 May 2020 


\section{References}

1. Johansson-Percival, A., He, B. \& Ganss, R. Immunomodulation of tumor vessels: it takes two to tango. Trends Immunol. 39, 801-814 (2018).

2. De Palma, M., Biziato, D. \& Petrova, T. V. Microenvironmental regulation of tumour angiogenesis. Nat. Rev. Cancer 17, 457-474 (2017).

3. Jourde-Chiche, $\mathrm{N}$. et al. Endothelium structure and function in kidney health and disease. Nat. Rev. Nephrol. 15, 87-108 (2019).

4. Motz, G. T. et al. Tumor endothelium FasL establishes a selective immune barrier promoting tolerance in tumors. Nat. Med. 20, 607-615 (2014).

5. Keir, M. E., Butte, M. J., Freeman, G. J. \& Sharpe, A. H. PD-1 and its ligands in tolerance and immunity. Annu. Rev. Immunol. 26, 677-704 (2008).

6. Chen, W. J. et al. Human umbilical vein endothelial cells promote the inhibitory activation of $\mathrm{CD} 4(+) \mathrm{CD} 25(+)$ Foxp3(+) regulatory T cells via PD-L1 Atherosclerosis 244, 108-112 (2016).

7. Pittet, C. L. Newcombe, J. Prat, A. \& Arbour, N. Human brain endothelial cells endeavor to immunoregulate CD8 T cells via PD-1 ligand expression in multiple sclerosis. J. Neuroinflamm. 8, 155 (2011)

8. Grabie, N. et al. Endothelial programmed death-1 ligand 1 (PD-L1) regulates CD8+ T-cell mediated injury in the heart. Circulation 116, 2062-2071 (2007)

9. Sun, C., Mezzadra, R. \& Schumacher, T. N. Regulation and Function of the PDL1 Checkpoint. Immunity 48, 434-452 (2018).

10. Schmittnaegel, $M$. et al. Dual angiopoietin-2 and VEGFA inhibition elicits antitumor immunity that is enhanced by PD-1 checkpoint blockade. Sci. Transl. Med. 9, pii: eaak9670 (2017).

11. Allen, E. et al. Combined antiangiogenic and anti-PD-L1 therapy stimulates tumor immunity through HEV formation. Sci. Transl. Med. 9, eakk9679 (2017).

12. Shen, G. et al. Anlotinib: a novel multi-targeting tyrosine kinase inhibitor in clinical development. J. Hematol. Oncol. 11, 120 (2018).

13. Zhou, A. P. et al. Anlotinib versus sunitinib as first-line treatment for metastatic renal cell carcinoma: a randomized phase ii clinical trial. Oncologist 24, e702e708 (2019).

14. Han, B. et al. anlotinib as a third-line therapy in patients with refractory advanced non-small-cell lung cancer: a multicentre, randomised phase II trial (ALTER0302). Br. J. Cancer 118, 654-661 (2018).

15. Liu, S., Qin, T., Jia, Y. \& Li, K. PD-L1 expression is associated With VEGFA and LADC patients' s.urvival. Front Oncol. 9, 189 (2019).

16. Fusi, A. et al. PD-L1 expression as a potential predictive biomarker. Lancet Oncol. 16, 1285-1287 (2015).
17. Chen, Y. et al. PD-L1 expression and tumor mutational burden status for prediction of response to chemotherapy and targeted therapy in non-small cell lung cancer. J. Exp. Clin. Cancer Res. 38, 193 (2019).

18. Sato, E. et al. Intraepithelial CD8+ tumor-infiltrating lymphocytes and a high CD8+/regulatory $\mathrm{T}$ cell ratio are associated with favorable prognosis in ovarian cancer. Proc. Natl Acad. Sci. USA 102, 18538-18543 (2005).

19. Cavalleri, T. et al. Combined low densities of FoxP3+ and CD3+ tumorinfiltrating lymphocytes identify stage $\|$ colorectal cancer at high risk of progression. Cancer Immunol. Res. 7, 751-758 (2019).

20. Fukumura, D., Kloepper, J., Amoozgar, Z., Duda, D. G. \& Jain, R. K. Enhancing cancer immunotherapy using antiangiogenics: opportunities and challenges. Nat. Rev. Clin. Oncol. 15, 325-340 (2018).

21. Juneja, V. R. et al. PD-L1 on tumor cells is sufficient for immune evasion in immunogenic tumors and inhibits CD8 T cell cytotoxicity. J. Exp. Med. 214 895-904 (2017).

22. Tang, H. et al. PD-L1 on host cells is essential for PD-L1 blockade-mediated tumor regression. J. Clin. Invest. 128, 580-588 (2018).

23. Tian, L. et al. Mutual regulation of tumour vessel normalization and immunostimulatory reprogramming. Nature 544, 250-254 (2017).

24. Huang, Y. et al. Improving immune-vascular crosstalk for cancer immunotherapy. Nat. Rev. Immunol. 18, 195-203 (2018).

25. Lane, R. S. et al. IFNgamma-activated dermal lymphatic vessels inhibit cytotoxic T cells in melanoma and inflamed skin. J. Exp. Med. 215, 3057-3074 (2018).

26. Lomas-Neira, J. et al. Novel role for PD-1:PD-L1 as mediator of pulmonary vascular endothelial cell functions in pathogenesis of indirect ARDS in mice. Front. Immunol. 9, 3030 (2018).

27. Wyss, J. et al. Stromal PD-1/PD-L1 expression predicts outcome in colon cancer patients. Clin. Colorectal Cancer 18, e20-20e38 (2019).

28. Vila, N. et al. Pretreatment of RPE cells with lutein can mitigate bevacizumab-induced increases in angiogenin and bFGF. Ophthalmic Res. $\mathbf{5 7}$ 48-53 (2017).

29. Tomita, Y. et al. Oncolytic herpes virus armed with vasculostatin in combination with bevacizumab abrogate glioma invasion via the CCN1 and AKT signaling pathways. Mol. Cancer Ther. 18, 1418-1429 (2019).

30. Jia, Y. et al. Effect of bevacizumab on the tight junction proteins of vascular endothelial cells. Am. J. Transl. Res. 11, 5546-5559 (2019). 